\title{
A $z=2.72$ Galaxy Gravitationally Lensed by the Cluster MS 1512+36: Reconstruction and Near-infrared Spectroscopy
}

\author{
Ivo Labbé \\ Leiden Observatory, P.O. Box 9513, NL-2300 RA Leiden, The \\ Netherlands \\ Marijn Franx \\ Leiden Observatory, P.O. Box 9513, NL-2300 RA, Leiden, The \\ Netherlands
}

\begin{abstract}
Using archival HST/WFPC2 optical data and near-infrared VLT/ISAAC spectroscopy, the properties of the Lyman-break galaxy cB58 are analyzed. The $\mathrm{z}=0.37$ galaxy cluster MS1512+36 lenses it into strongly magnified arcs with ample substructure. The data enables an accurate reconstruction of the source, yielding milliarcseconds resolution; an unparalleled view of the central $\mathrm{kpc}$ of the $\mathrm{z}=2.72$ starburst galaxy.
\end{abstract}

\section{Introduction}

The galaxy population selected with the Lyman-break technique illustrates two limitations on present optical studies: the objects are very faint, hampering spectrocopy, and very compact, requiring at least HST resolution to resolve the inner parts. In the case of cB58, strong gravitational lensing by the cluster MS1512+36 helps to overcome these obstacles. Here we summarize the reconstruction of the source based on HST/WFPC2 images together with near-infrared VLT/ISAAC spectroscopy. The aim is to study the detailed morphology and the resolved colors of the lensed galaxy, using its structure to obtain the best possible lensmodel. The spectra will clarify the nature of the emission and supply dynamical information.

\section{Observations and modelling}

We used archival HST/WFPC2 images of the cluster MS1512+36 (program 6832, June 1997) in two broad-band filters; 2.9 (4.8) hrs in $\mathrm{V}_{555}\left(\mathrm{I}_{814}\right)$ reaching 24.0 (23.8) $\operatorname{mag}_{A B} \operatorname{arcsec}^{-2}(1 \sigma$ limit). The raw frames are dithered and interlacing leads to a $0.0325^{\prime \prime}$ pixel $^{-1}$ scale. The VLT/ISAAC observations (program 60.A-9021(A) \& 263.O-0705(A), spring 1999) were conducted in SWS1-LR mode. The $1^{\prime \prime}\left(0.6^{\prime \prime}\right)$ longslit spectrum in $\mathrm{H}(\mathrm{K})$ totalled $1.4(1.0)$ hrs with a 5 (7) $\AA$ pixel ${ }^{-1}$ dispersion. 

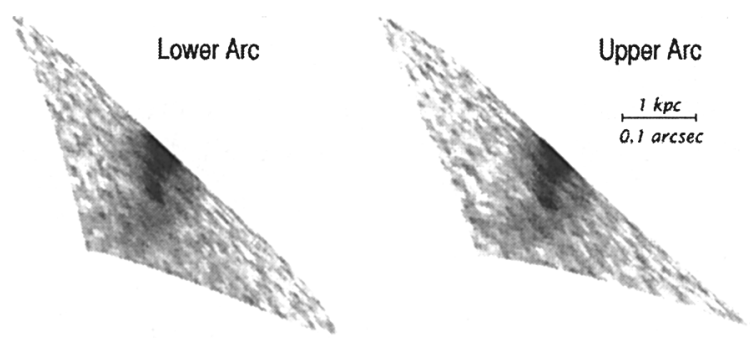

Figure 1. The two deprojected $\mathrm{V}_{555}$ images of the giant arc (2 - 10 milliarcsecond pixel ${ }^{-1}$ ) showing several sites of star formation.

The gravitional lens is modelled as a superposition of elliptical isothermal potentials, where the parameter values are determined by minimizing a $\chi^{2}$ statistic between the predicted positions in the source plane of corresponding features in the fold-arc (Seitz et al. 1998).

\section{Results}

As shown in Figure 1, the dramatic detail in the reconstruction permits the division of cB58 in distinct components: diffuse emission interspersed with compact knots, measuring $30-80 \mathrm{~h}_{50}^{-1} \mathrm{pc}$ in an Einstein-de Sitter cosmology. The high surface brightness of the compact regions, $4-9 \times 10^{12} \mathrm{~L}_{\odot} \mathrm{kpc}^{-2}$, reminds of local starbursts which show two modes of star formation: prominent stellar cluster formation and diffuse stellar light. The ratios of the nebular lines to the forbidden lines in the ISAAC spectra support this picture. The rest-frame far-UV colors are spatially resolved; the knots are bluest and the envelope is redder, with a difference in spectral slope of $\Delta \beta \approx 0.5$. Exclusively attributed to aging, this means the surroundings look $1 \mathrm{Gyr}(100 \mathrm{Myr})$ older for a continuous (instantaneous) star formation model. The envelope flux can be produced by $\sim$ $1.4 \times 10^{8} \mathrm{~A}$ stars; thus the old stellar mass fraction is small. From ISOCAM data, Bechtold, Yee, \& Elston (1997) already had put strong limits on the $1 \mathrm{~h}_{50}^{-1}$ Gyr old mass fraction $(\$ 10 \%)$. Otherwise, if caused by differential extinction the color difference can be produced if the knots are less extinct by about 0.6 mag for a Calzetti attenuation law. Finally, the $\mathrm{H} \alpha$ linewidth $\left(\sigma \approx 160 \mathrm{~km} \mathrm{~s}^{-1}\right)$ at face value gives a tentative determination of the virial mass of about $1.5 \mathrm{x}$ $10^{10} \mathrm{M}_{\odot}$, comparable to the Milky Way bulge.

\section{References}

Bechtold, J., Yee, H. K. C., \& Elston, R., E. 1997, ApJ, 477, L29

Seitz, S., Saglia, P. R., Bender, R., Hopp, U., Belloni, P., \& Ziegler, B. 1998, MNRAS, 298, 945-965 